\title{
Surgery in spontaneous intracerebral hemorrhage- a series analysis
}

\begin{abstract}
The medical and or surgical treatment of spontaneous ICH appears to be a matter of controversy. Vascular malformations, aneurysms and drug abuse are causative factors for spontaneous ICH among the young adults while hypertension (most important and prevalent risk factor), tumors, vasculopathy, coagulopathy and cerebral amyloid angiopathy are causes among older adults. Most of the studies show no significant difference between intense medical therapy or surgery.

A prospective analysis was done of patients who underwent surgical intervention for spontaneous ICH. The indications for surgery were a spontaneous ICH confirmed by CT scan, Glasgow Coma Scale (GCS) $>4$ and volume of clot $>30$ cc. Surgical techniques included evacuation by open craniotomy, hemostasis under microscope and adequate decompression (10-15 cm flap removal and duraplasty). Postoperatively, all patients were given multimodality supportive care. A total of 30 patients underwent surgical intervention. They were of age between 7 to 70 years. The causes of lesional haemorrhages were vascular malformation, vasculitis, aneurysm etc. The mortality rate was $40 \%$, much lower than in earlier surgical series, where it is documented as $56 \% 0^{1,2}$ The survival rate among those with lesional hemorrhage $(90 \%)$ was much higher than those with primary hemorrhage $(47 \%)$. Lobar hemorrhage was also found to be linked with better survival rates $(80 \%)$ than the basal ganglia hemorrhage $(41.6 \%)$. Age also was found to be a prognostic factor. Survival was found to be related inversely with age.
\end{abstract}

Early surgery combined with multimodality medical management has definite positive role in the treatment of patients with spontaneous ICH, especially young patients with lobar and lesional hemorrhage.

Keywords: spontaneous intracerebral hemorrhage, systemic hypertension, arteriovenous malformation, cerebral vasculitis, cerebral amyloid angiopathy, cerebral artery aneurysm, decompressive craniectomy, cerebral vasospasm, cerebroprotective therapy
Volume 2 Issue 4 - 2015

\author{
Ajaya Kumar A,' Joanna Sara Valson² \\ 'Neurosurgeon, Mar Gregorios Memorial Muthoot Medical \\ Centre, India \\ ${ }^{2}$ Research assistant, Mar Gregorios Memorial Muthoot Medical \\ Centre, India
}

Correspondence: Dr. Ajaya Kumar A., M.B.B.S., M.S., D.N.B., M.Ch., Neurosurgeon, Mar Gregorios Memorial Muthoot Medical Centre, Kozhencherry, Kerala, India, Email drajayakumara@gmail.com

Received: May 06, 2015 | Published: July 15, 2015

\section{Background}

Spontaneous Intra-cerebral Hemorrhage $(\mathrm{ICH})$ is considered a great public health problem, accounting for $9-25 \%$ of all strokes, ${ }^{1} 20-30 \%$ of all strokes among Asian populations. ${ }^{2}$ The medical and or surgical treatment of spontaneous ICH appears to be a matter of controversy. ${ }^{3}$ Spontaneous ICH can be distinguished as Primary and Secondary or Lesional and Non-lesional (based on the causative factor). It is evidenced that vascular malformations, aneurysms and drug abuse are causative factors for spontaneous ICH among the young adults while hypertension (most important and prevalent risk factor),${ }^{4}$ tumors, vasculopathy, coagulopathy and cerebral amyloid angiopathy are causes among older adults. Other risk factors include low cholesterol levels, heavy alcohol intake and cigarette smoking. Among children, leukemia is found to be a significant cause.

In relation to the treatment of spontaneous $\mathrm{ICH}$, most of the studies show no significant difference with intense medical therapy, surgery or combined surgery and intense medical therapy. However, the International STICH (Surgical Trial in Intracerebral Hemorrhage) produced results that surgery within 96 hours of ictus was associated with statistically insignificant absolute benefit of $2.3 \% .^{5}$

Treatment strategies could comprise of medical or surgical interventions. Medical therapy largely comprises of blood pressure management, intracranial pressure management, hemostatic therapy (using recombinant activated factor VII/ FFP) and neuro- protective agents. However, there is a risk for the penumbra of progressive tissue damage immediately surrounding hematoma. It is due to mechanical injury caused by increased pressure, decreased cerebral blood flow, inflammation due to accumulated proteins and protease induction. Thus, to reduce this secondary injury, surgical intervention is necessary. Surgical intervention includes Craniotomy (where complete evacuation is done under vision) in case of lobar hematomas/exploration of vascular lesions and Aspiration (using a burr hole, stereotactic or endoscopic measures) of smaller hematomas, producing desirable results. ${ }^{4,6}$

The STICH trial produced results showing that patients with hematomas extending to within one centimetre of cortical surface had a trend toward more favourable outcome. Also, patients with lobar haemorrhages and GCS of 9-12 also had better outcomes. Patients, whom the local investigator felt would most likely benefit from emergency surgery, were not enrolled in the trial. ${ }^{7}$

\section{Method}

A prospective analysis was done of patients who underwent surgical intervention for spontaneous ICH. They were in non-controversial group where surgery was absolutely indicated by the volume of clot and mass effect. These patients were studied prospectively -age, G.C.S, neurological deficits, CT/MRI findings, surgery, postoperative period, complications and outcome. 
Initial neurological status at the time of admission was assessed using Glasgow Coma Scale (GCS). The time of onset of symptoms was recorded based on interview with the patient and family. The diagnosis of ICH was based on the signs observed and the symptoms determined, along with confirmed CT scans. CT and/ or MR angiography was performed on emergency basis or after recovery to exclude vascular pathology. The size of the hematoma was determined by $1 / 2 \mathrm{ABC}$, where $\mathrm{A}, \mathrm{B}$ and $\mathrm{C}$ stands for maximum dimensions of hematoma. The patient was admitted to neurosurgery only after discussion with the patient's family or first-degree relatives about guarded outcome and chance of vegetative state.

The indications for surgery were a spontaneous ICH confirmed by CT scan, Glasgow Coma Scale (GCS) $>4$ and volume of clot $>30 \mathrm{cc}$. Patients above 70 years and those with significant IVH were excluded.

\section{Surgical approach}

Following informed consent, surgery was undertaken within 24 hours of admission with standard neurosurgical techniques. The type of surgery was individualised and was based on the size and site of ICH. Surgical techniques included evacuation by open craniotomy, hemostasis under microscope and adequate decompression (10-15 cm flap removal and duraplasty). Microscope helped in getting adequate hemostasis with isocool cautery, surgical and gelfoam. Large flap removal aided in reducing the edema. Definitive treatment of lesion (excision of AVM, clipping of aneurysm) was also done along with evacuation of clot.

\section{Multimodality medical approach}

Postoperatively, all patients were given multimodality supportive care. Physician, nephrology, cardiology and critical care specialist were involved. Close monitoring of neurological status, level of consciousness, vital signs were done. Normotension, ventilation under sedation to reduce $\mathrm{pCO} 2$ to $30 \mathrm{mmHg}$ and mild hypothermia $(35 \mathrm{C})$ were given the priority. Intense anti-edema and cerebral protectant therapy was administered during the acute phase. Mannitol reduces edema and improves cerebral perfusion. Thiopentone reduces brain metabolism. Nimodipine reduces vasospasm. Citicoline has membrane stabilising action. Coagulopathy was corrected by FFP and platelet concentrate. Measures included IV fluid therapy, prophylaxis for deep vein thrombosis, physical therapy, infection control and preventive techniques and early nutritional support. Postoperative CT scan was done once the patient was stable. Ventilation was reduced once brain edema started settling. For conscious patients incentive spirometry was given for preventing hypercapnia and pulmonary complications. Limb physiotherapy was started early.

\section{Measurement of outcome}

Outcome measurement was done using Glasgow Outcome Scale (GOS) at the end of six months postoperatively. Neurological status of each patient was also documented.

\section{Results}

A total of 30 patients underwent surgical intervention. They were of age between 8 to 70 years. GCS score ranged between 5 and13. The hematoma volume extended from 30 to $90 \mathrm{cc}$. The time of surgery was within 12-72 hours after onset of ICH. Major complications addressed were cerebral edema, rebleed, vasospasm, hypostatic pneumonia, renal failure, hepatic failure, pulmonary embolism and atrial fibrillation (Table $1 \& 2$ ).

The prognosis of the patients was assessed using Glasgow Outcome scale. The results are summarised in Table 3.
Table I Table showing age groups and rates of survival

\begin{tabular}{lll}
\hline Age group & $\mathbf{N}(\%)$ & Survival: $\mathbf{n}(\%)$ \\
\hline Up to $20 y r$ & $2(6.6 \%)$ & $2(100 \%)$ \\
$20-40 y r$ & $5(16.6 \%)$ & $4(80 \%)$ \\
$40-60 y r$ & $16(53.3 \%)$ & $9(56.2 \%)$ \\
Above $60 y r$ & $7(23.3 \%)$ & $3(42.9 \%)$ \\
\hline
\end{tabular}

Table 2 Table showing type of hematoma and rates of survival

\begin{tabular}{llll}
\hline Category & Type & N (\%) & Survival: n (\%) \\
\hline \multirow{2}{*}{ Lesional } & Lobar haemorrhage & $8(26.6)$ & $7(87.5)$ \\
& Basal Ganglia hemorrhage & $2(6.6)$ & $2(100)$ \\
\multirow{2}{*}{ Non-lesional } & Primary lobar hemorrhage & $7(23.3)$ & $5(71.4)$ \\
& Primary Basal ganglia hemorrhage & $10(33.3)$ & $3(30)$ \\
Infarct & Hemorrhagic Infarct & $3(10)$ & $1(33.3)$ \\
Total & & 30 & $18(60)$ \\
\hline
\end{tabular}

Table 3 Table showing outcome measures after 6-month period

\begin{tabular}{|c|c|c|}
\hline $\begin{array}{l}\text { Glasgow } \\
\text { outcome scale }\end{array}$ & Condition & $\mathbf{N}(\%)$ \\
\hline Death & $\begin{array}{l}\text { Severe injury or death without } \\
\text { recovery of consciousness }\end{array}$ & $12(40)$ \\
\hline $\begin{array}{l}\text { Persistent } \\
\text { vegetative state }\end{array}$ & $\begin{array}{l}\text { Severe damage with prolonged state of } \\
\text { unresponsiveness and a lack of higher } \\
\text { mental functions }\end{array}$ & I (3.3) \\
\hline Severe disability & $\begin{array}{l}\text { Severe injury with permanent need for } \\
\text { help with daily living }\end{array}$ & $3(10)$ \\
\hline Moderate disability & $\begin{array}{l}\text { No need for assistance in everyday life, } \\
\text { employment is possible but may require } \\
\text { special equipment }\end{array}$ & $5(16.7)$ \\
\hline Low disability & $\begin{array}{l}\text { Light damage with minor neurological } \\
\text { and psychological deficits, good } \\
\text { recovery }\end{array}$ & $9(30)$ \\
\hline
\end{tabular}

The outcome measures among the survivors showed that $50 \%$ survived with low disability, $27.8 \%$ with moderate disability, $16.6 \%$ with severe disability and only one $(5.6 \%)$ declined into persistent vegetative stage.

\section{Prototype of each category of ICH}

\section{Case 1: Lesional Lobar hemorrhage due to AVM}

A 7 year old boy was admitted with GCS score $\mathrm{E}_{2} \mathrm{M}_{5} \mathrm{~V}_{1}$ presenting with right hemiplegia. He was diagnosed as having Left frontoparietal hemorrhage (Figure 1.1). Decompressive craniectomy and evacuation (Figure 1.2) was done and the patient was ventilated for 3 days. MR angiography showed AVM fed by branches of MCA (Figure 1.3), draining into the superior sagittal sinus and deep veins-SpetzlerMartin Grade 3 (Figure 1.4). AVM was excised. Postoperatively after 6 months, CT angiography showed medial recurrence, draining into the deep venous system (Figure 1.5). Complete excision was done (Figure 1.6) and the boy recovered with no deficits.

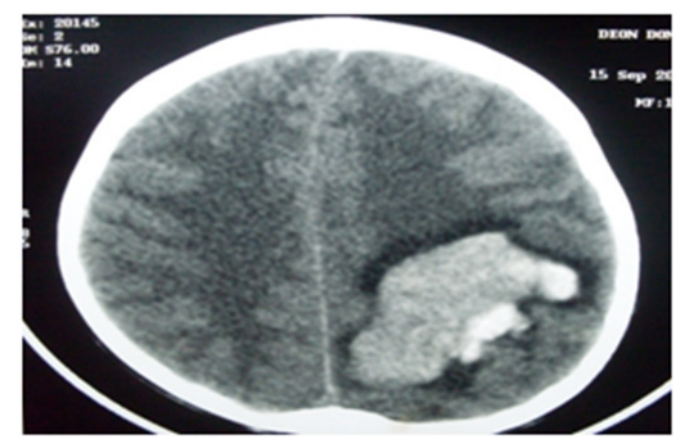

Figure I.I CT Scan showing left fronto-parietal hemorrhage. 


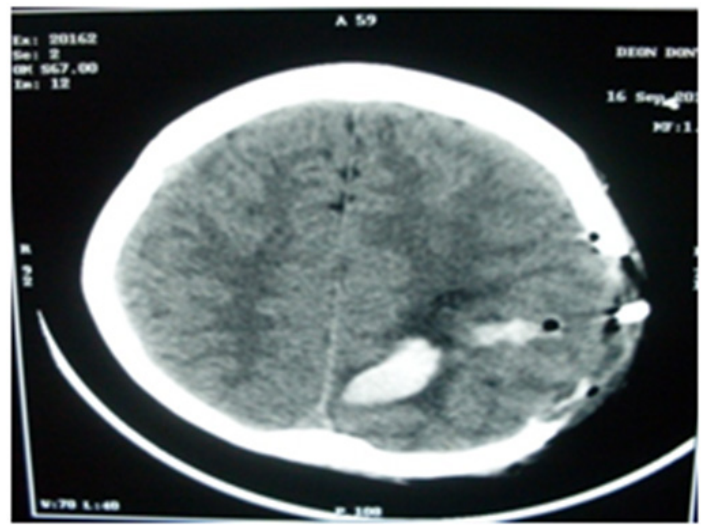

Figure I.2 CT scan following decompressive craniectomy and evacuation.

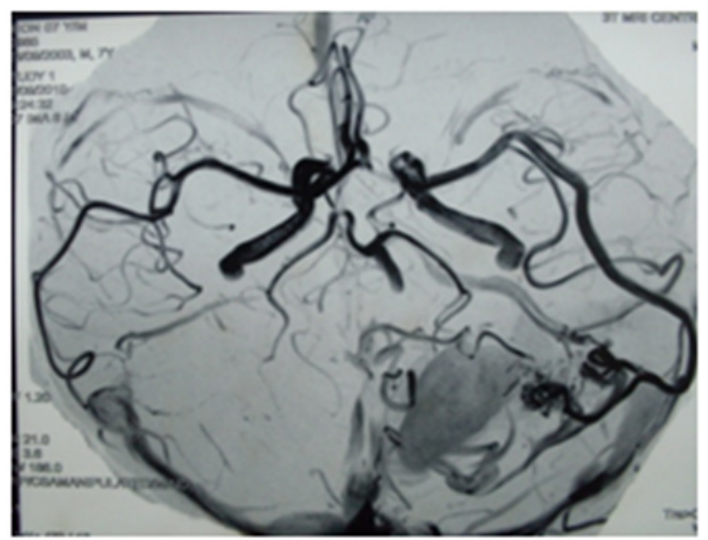

Figure I.3 MR angiography showing AVM fed by branches of MCA.

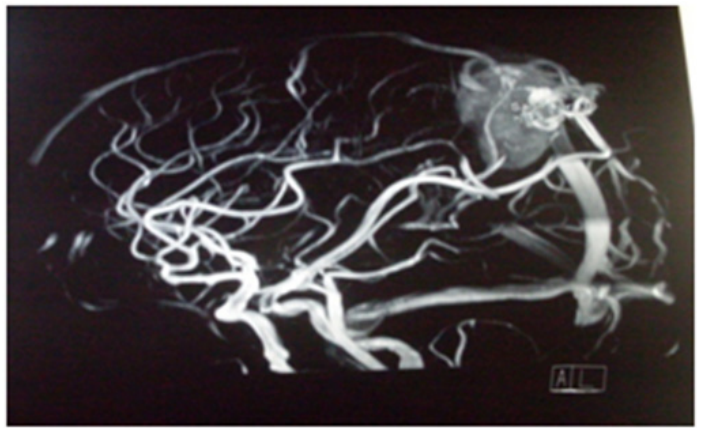

Figure I.4 MR angiography showing AVM draining into superior sagittal sinus and deep veins.

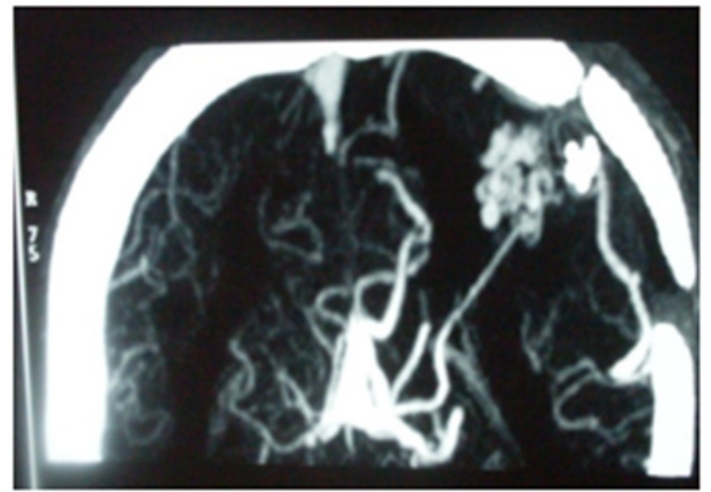

Figure I.5 CT scan showing medial recurrence, draining into the deep venous system.

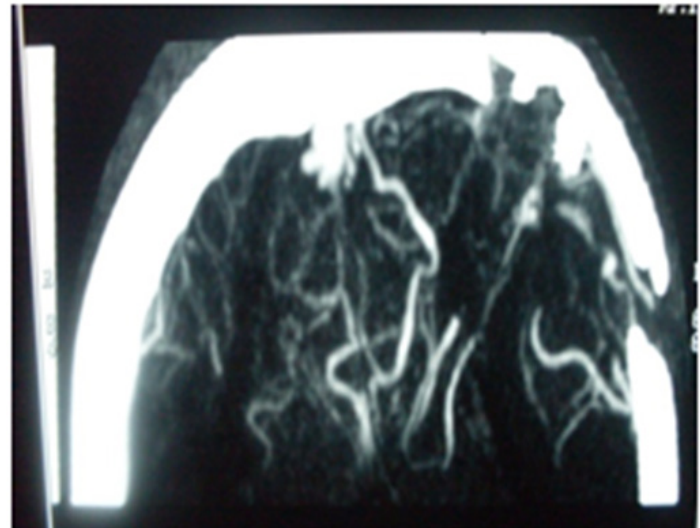

Figure I.6 CT scan showing complete excision of ICH.

\section{Case 2: Lesional Lobar hemorrhage due to vasculitis}

An 8 year old boy presented with dilated left pupils and a GCS score of $8\left(\mathrm{E}_{2} \mathrm{M}_{5} \mathrm{~V}_{1}\right)$. He was diagnosed as having left temporoparietal hemorrhage (Figure 2.1). MRI with contrast showed minimal enhancement and large bleed with varying stages of bleed (Figure $2.2 \& 2.3$ ). Temporo-parietal craniectomy (Figure 2.4) was done to completely evacuate the clot (Figure 2.5). Histopathology results confirmed small vessel vasculitis (Figure 2.6). The boy recovered with no significant deficits.

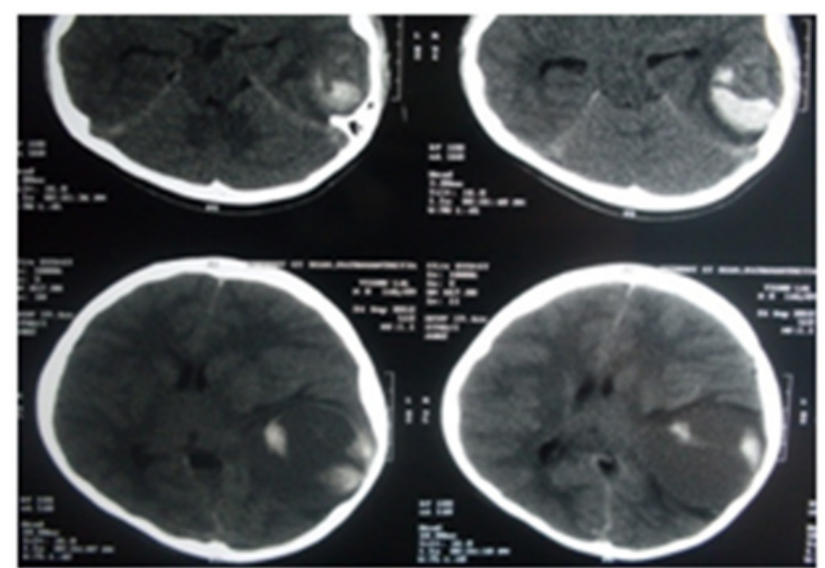

Figure 2.I CT Scan showing left temporo-parietal hemorrhage.

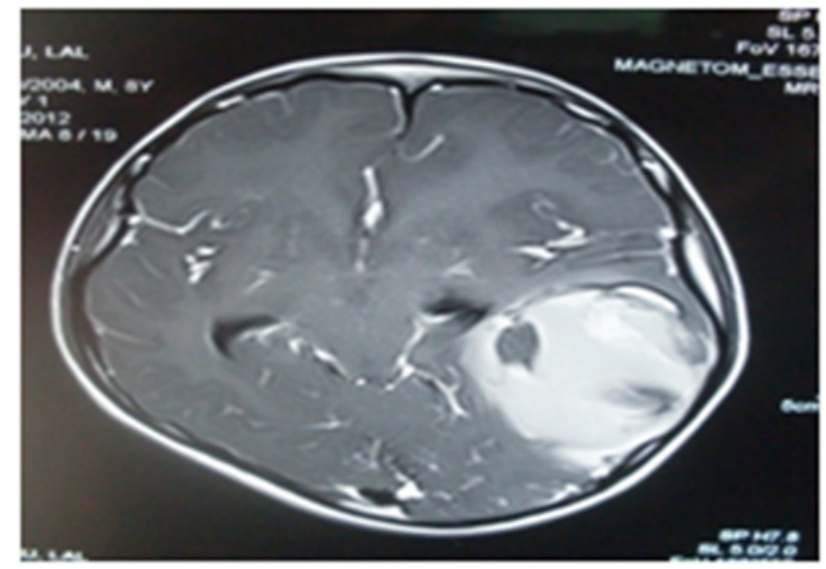

Figure 2.2 MRI contrast showing minimal enhancement of the bleed. 


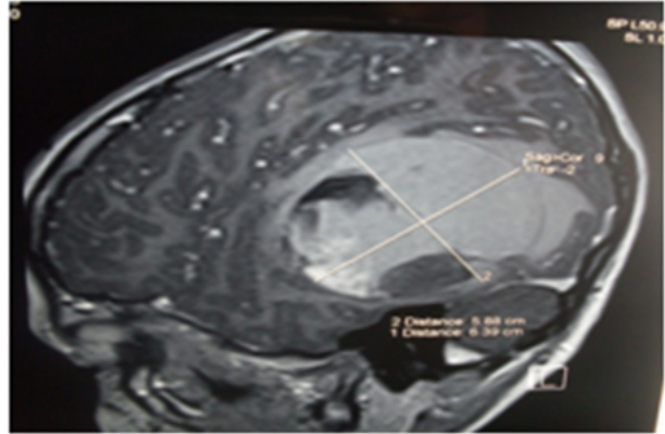

Figure 2.3 MRI contrast showing large bleed with varying stages.

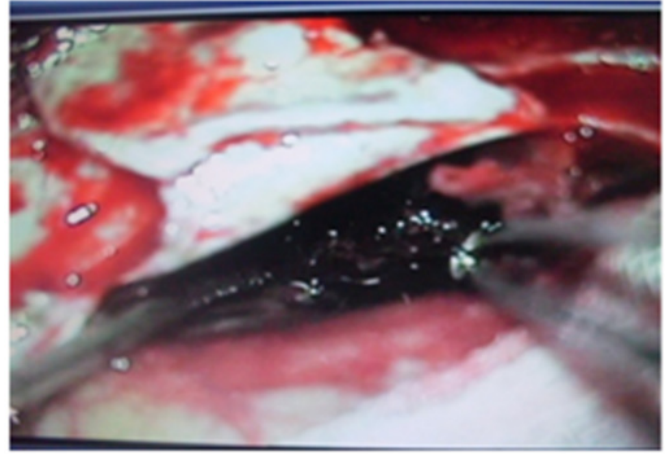

Figure 2.4 Clots, debris and vascular tissue seen during temporo-parietal craniectomy.

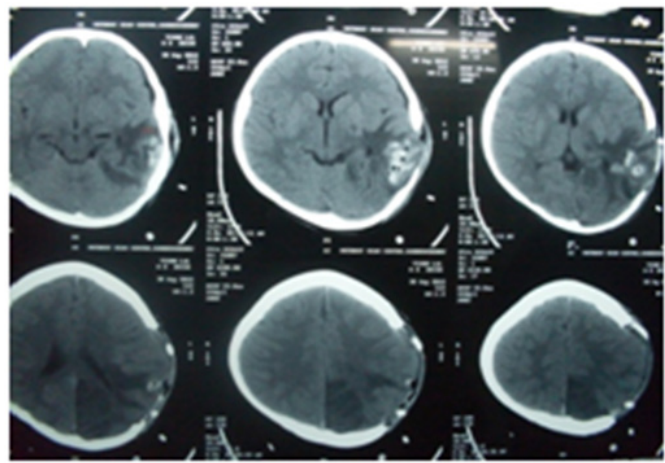

Figure 2.5 CT scan showing complete evacuation post-operatively.

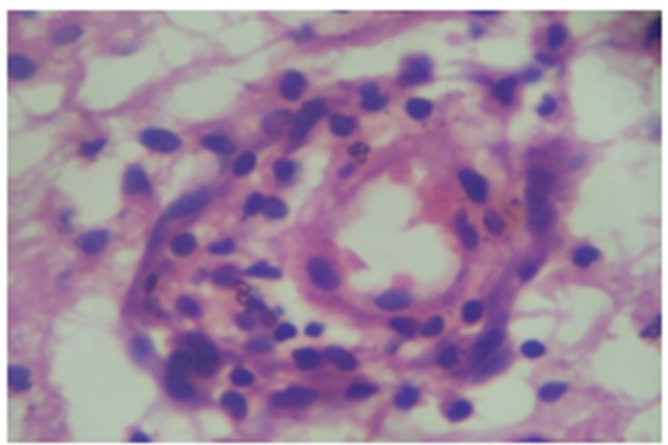

Figure 2.6 Histopathology specimen showing vessel with infiltration of polymorphs.

Case 3: Lesional Lobar hemorrhage due to aneurysm

A 40 year old female presented with left pupil dilation and was diagnosed as having frontal hematoma (Figure 3.1). MR angiography showed anterior cerebral artery aneurysm bleed (Figure 3.2) and coronal projection showed the extent of hematoma (Figure 3.3). 3-D CT with angiography was also done for planning (Figure 3.4). Evacuation of hematoma and clipping of aneurysm was done (Figure

3.5 ) and the patient recovered without deficits.

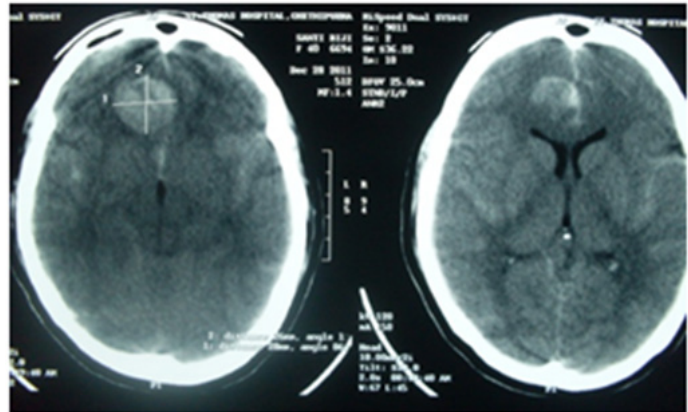

Figure 3.I CT Scan showing frontal hematoma.

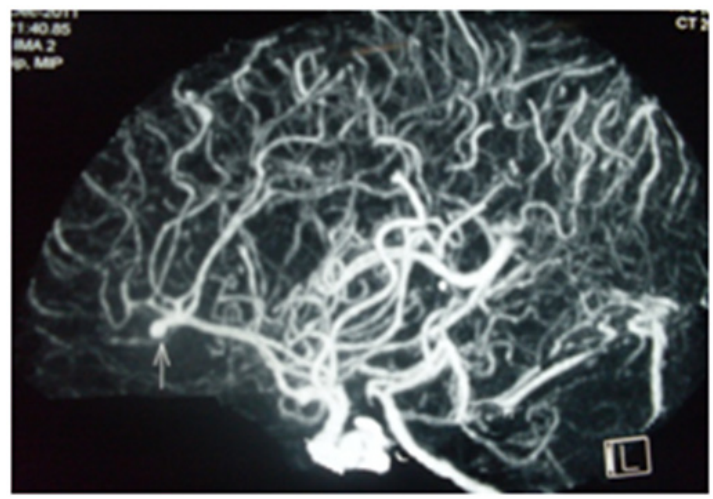

Figure 3.2 MR Angiography showing anterior cerebral artery aneurysm bleed.

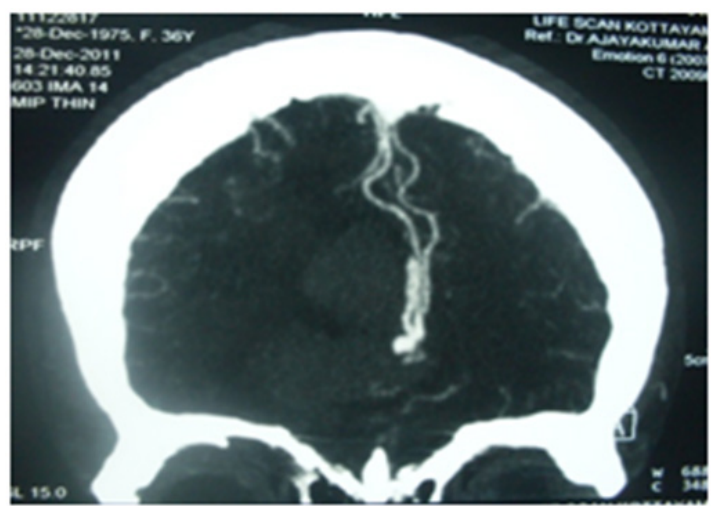

Figure 3.3 Coronal projection showing the extent of hematoma.

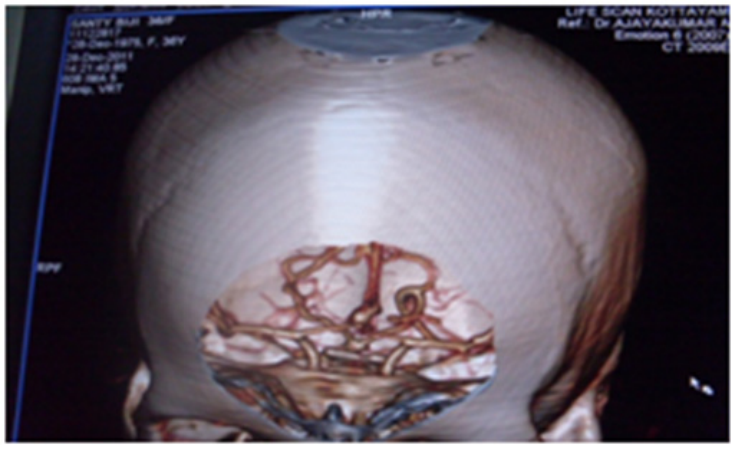

Figure 3.4 3D CT scan with angiography showing the aneurysm 


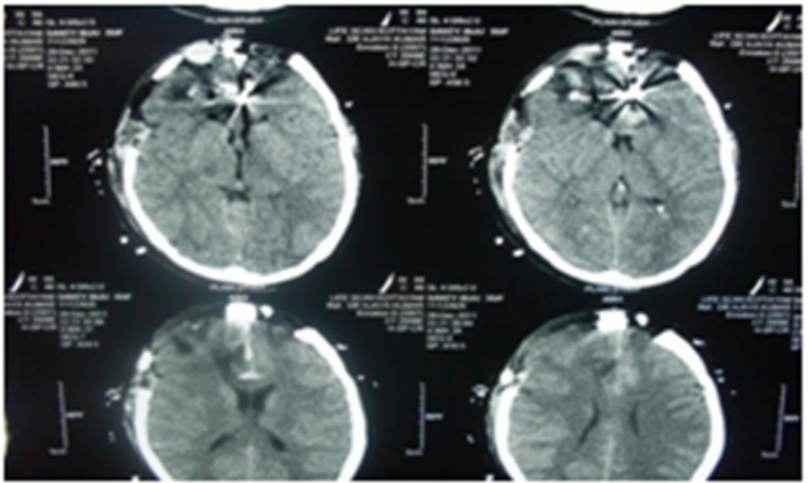

Figure 3.5 CT scan showing evacuation of hematoma.

Case 4: Lesional Lobar hemorrhage with vasospasm

A 60 year old male presented with loss of consciousness and left-side weakness. He was diagnosed as having right temporal and putaminal bleed extended up to temporal floor (Figure 4.1). MR angiography showed vasospasm of M1 (Figure 4.2) and coronal projection showed the same (Figure 4.3). Evacuation was done. Active bleeding was noticed from perforator (Figure 4.4), which was cauterised with Isocool cautery. Postoperatively CT Scan showed complete evacuation(Figure 4.5) and MR angiography showed vasospasm relieved and duplication of M1 (Figure 4.6) and the patient was relieved of weakness.

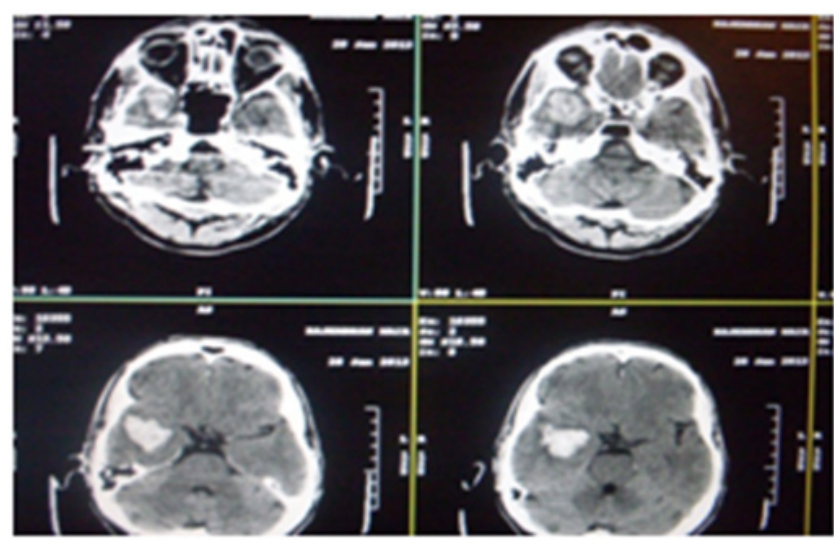

Figure 4.I CT Scan showing right temporal and putaminal bleed extended to temporal floor.

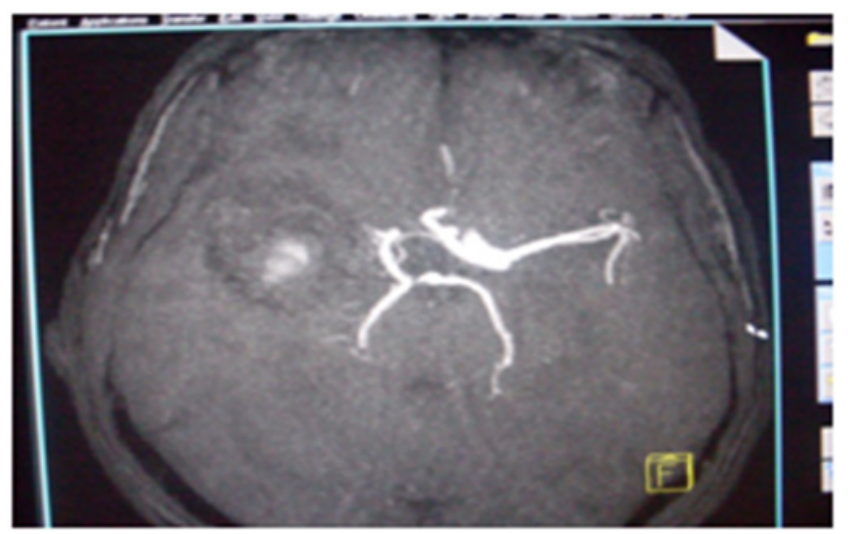

Figure 4.2 MR Angiography showing vasospasm of MI.

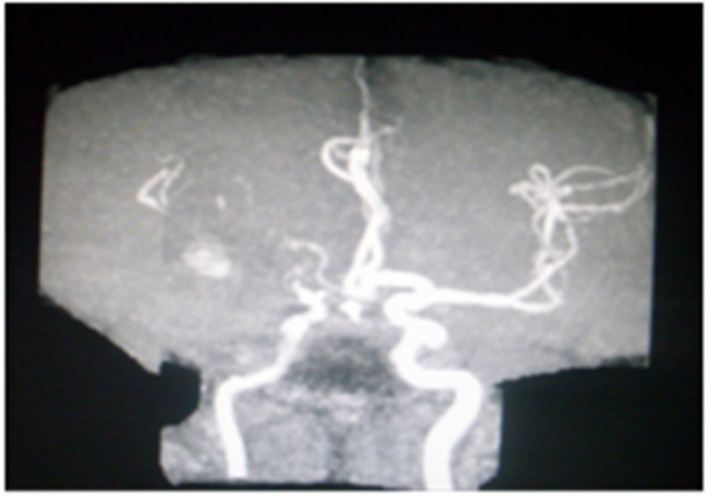

Figure 4.3 Coronal projection showing the extent of vasospasm.

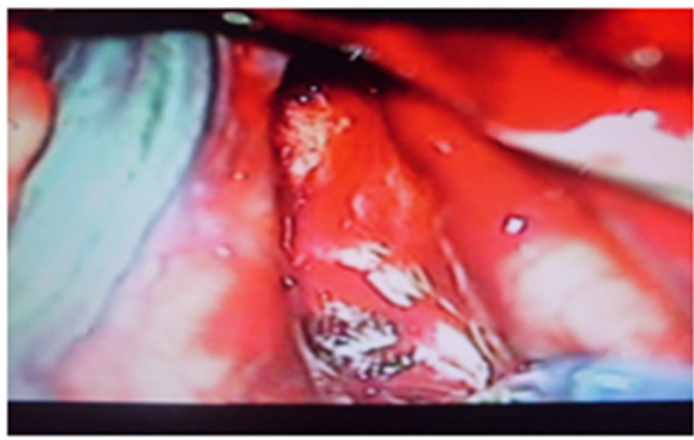

Figure 4.4 Active bleeding noticed from perforator.

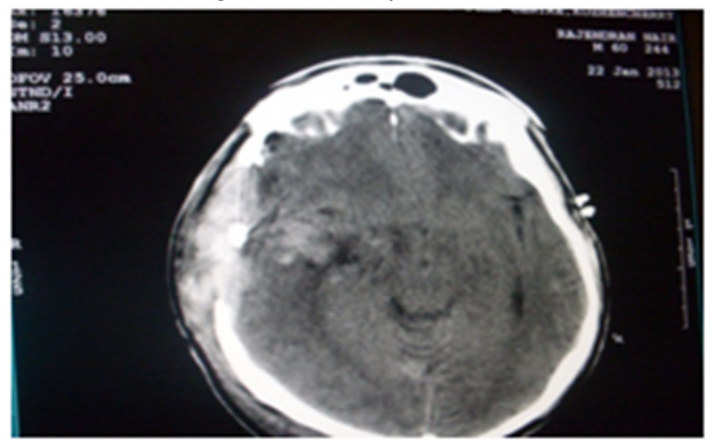

Figure 4.5 CT scan showing complete evacuation post-operatively.

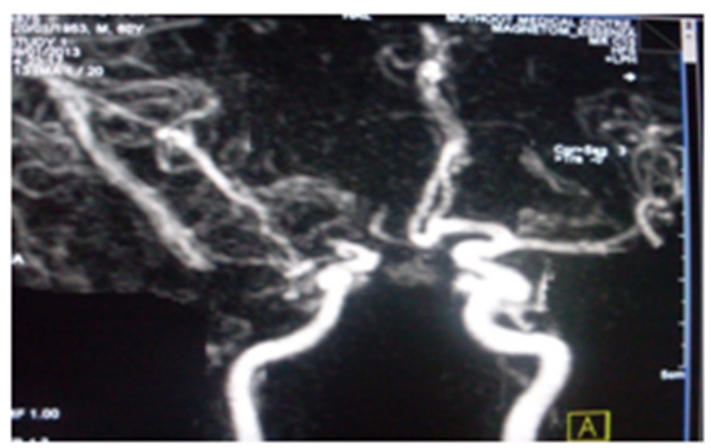

Figure 4.6 MR Angiography showing vasospasm relieved and MI duplication.

\section{Case 5: Primary Lobar hemorrhage}

A 52 year old male presented with right hemiparesis and GCS score 10. He was diagnosed as having left parietal hemorrhage (Figure 5.1). Decompression and duraplasty with G-patch was done (Figure 5.2) and the patient was relieved of weakness. 


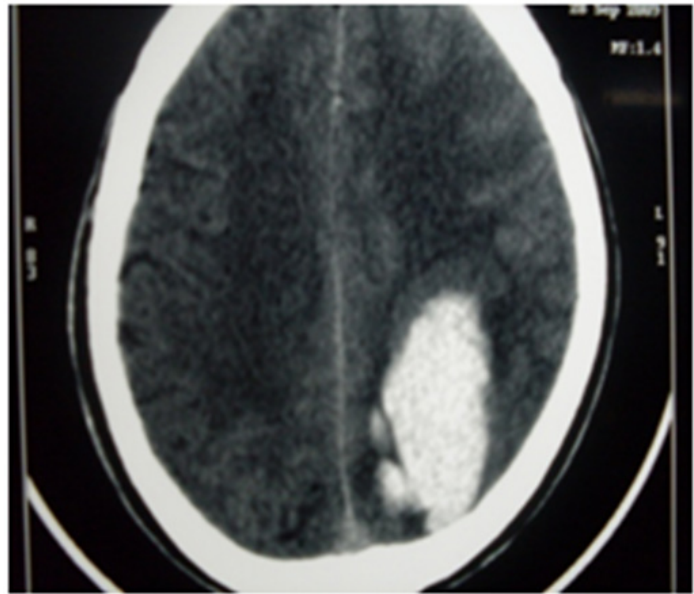

Figure 5.I MR Angiography showing vasospasm relieved and MI duplication.

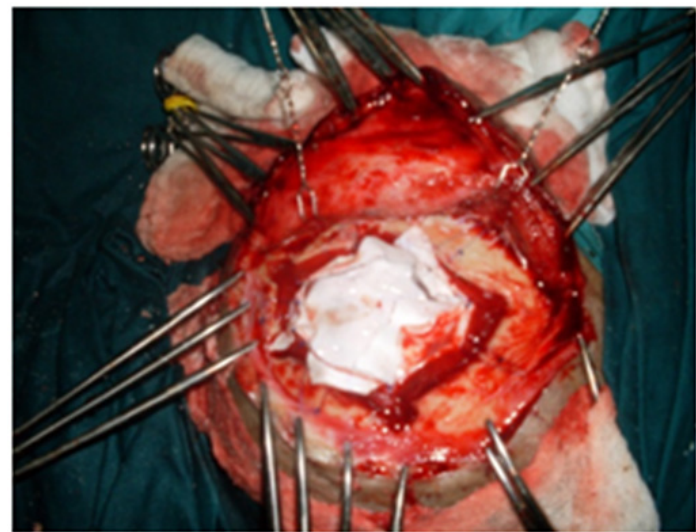

Figure 5.2 Decompression and duraplasty done with G-patch.

Case 6: Lesional basal ganglia hemorrhage due to vasculitis

A 30 year old male presented with left hemiparesis and GCS score of $10\left(\mathrm{E}_{3} \mathrm{M}_{5} \mathrm{~V}_{2}\right)$. He was diagnosed as having right basal ganglia bleed (Figure $6.1 \&$ 6.2). Complete evacuation was done (Figure 6.3). HPR came as vasculitis. The patient achieved total improvement postoperatively.

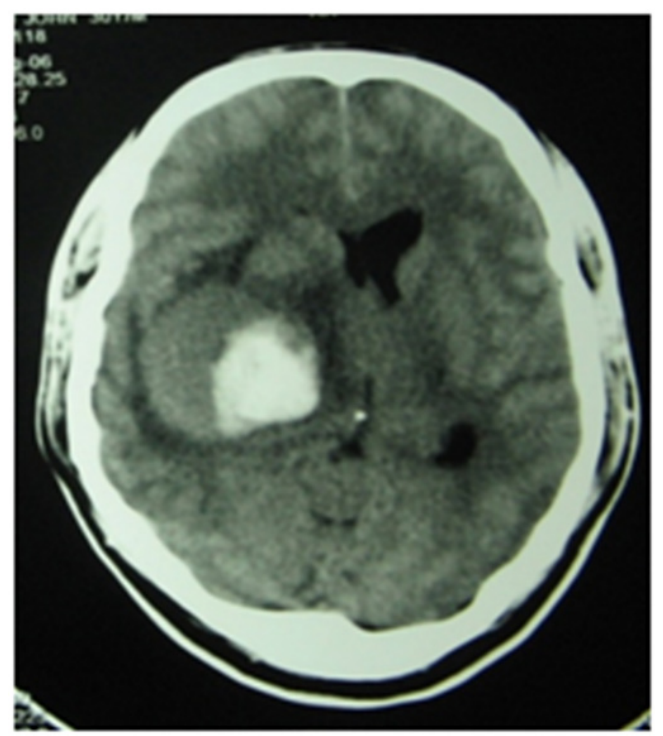

Figure 6.I CT Scan showing right basal ganglia bleed.

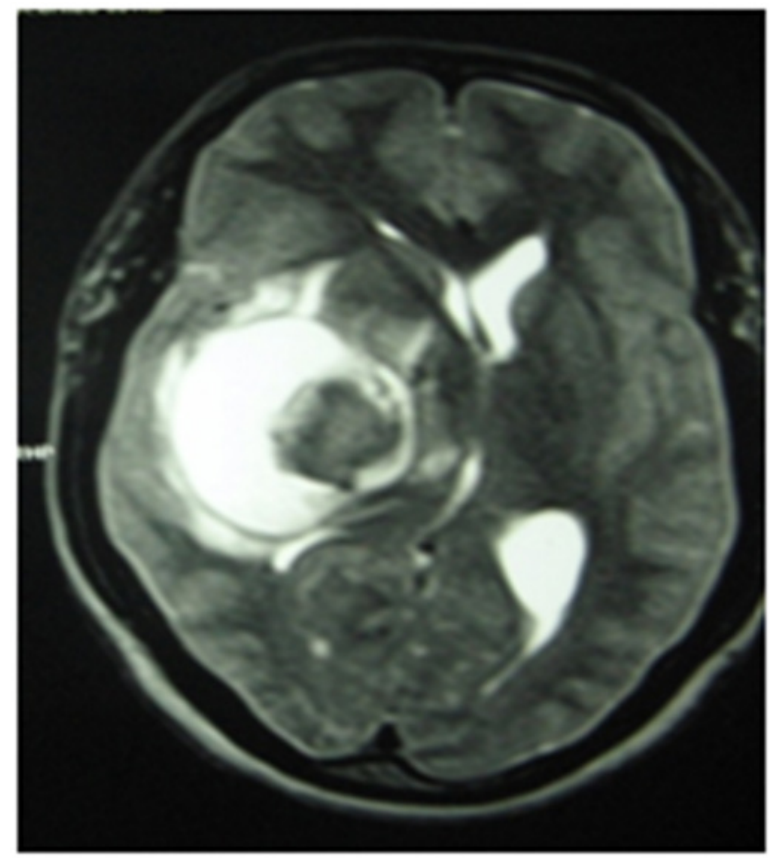

Figure 6.2 MRI scan showing right basal ganglia bleed.

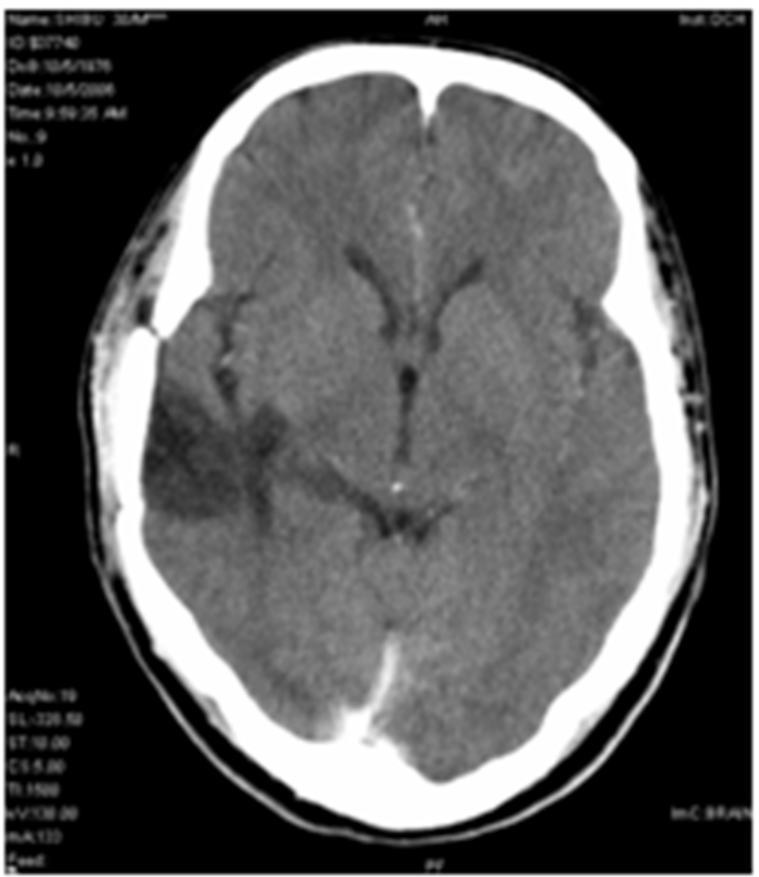

Figure 6.3 CT scan showing complete evacuation of bleed.

Case 7: Lesional basal ganglia hemorrhage due to vascular malformation

A 67 year old man presented with GCS score of $5\left(E_{1} M_{3} V_{1}\right)$. He had headache and left paresis one year ago. MRI showed suspicious hyper-intensity right basal ganglia bleed (Figure 7.1). Decompressive craniectomy was done and vascular malformation seen in bed was excised (Figure 7.2). Postoperative CT angiogram showed small arteriolar tufts from middle cerebral artery (MCA) (Figure 7.3). The patient recovered after 8 months. 


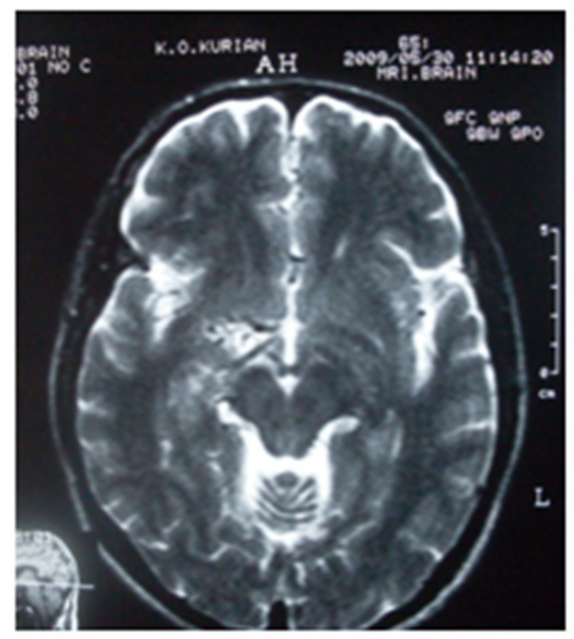

Figure 7.I MRI Scan showing right basal ganglia bleed.

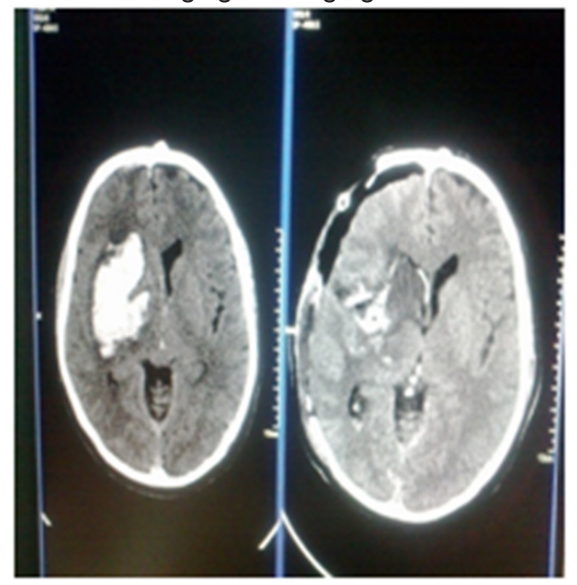

Figure 7.2 CT scan showing complete excision of vascular malformation.

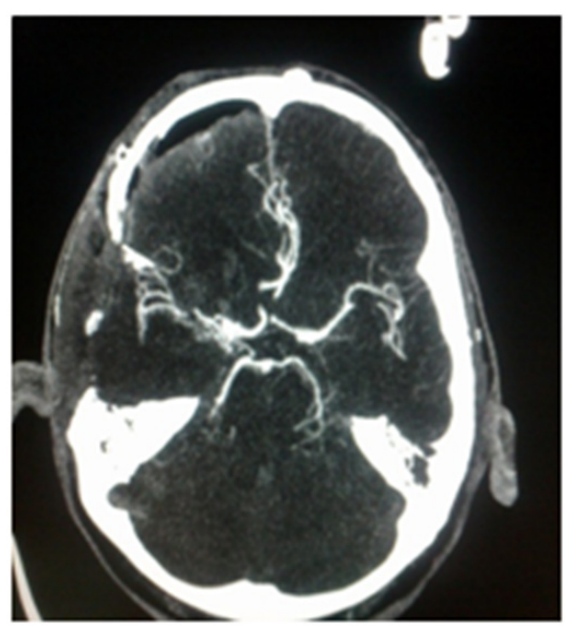

Figure 7.3 Postoperative CT angiogram showing arteriolar tufts from MCA.

Case 8: Primary basal ganglia hemorrhage

A 52 year old man presented with GCS score of $5\left(E_{1} M_{3} V_{1}\right)$ and was diagnosed as having left basal ganglia bleed. CT angiography showed spasm of M1 (Figure 8.1). Decompressive craniectomy was done to evacuate the clot (Figure 8.2). The patient was ventilated but he declined into vegetative stage.

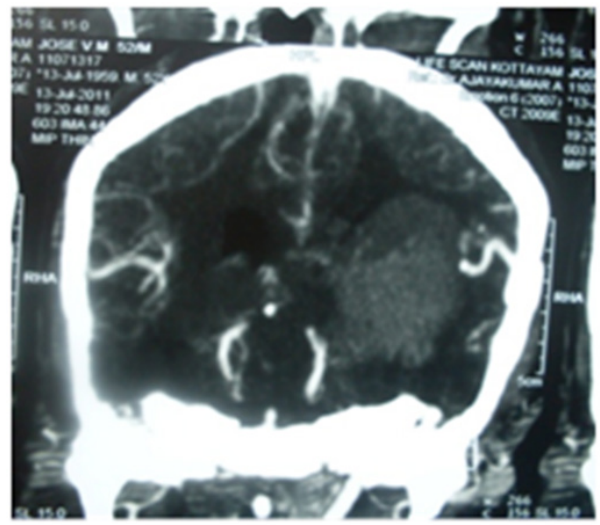

Figure 8.I CT angiography showing spasm of MI.

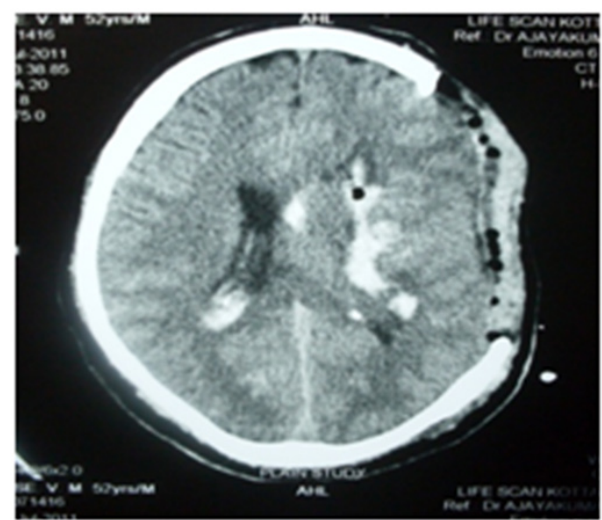

Figure 8.2 CT scan showing excision of clot.

\section{Case 9: Hemorrhagic Infarct}

A 40 year old male presented with seizures, left hemiplegia and a GCS score of $\mathrm{E}_{2} \mathrm{M}_{5} \mathrm{~V}_{2}$. He was diagnosed as having right temporoparietal hemorrhage (Figure 9.1). He improved after surgery but deteriorated after 10 days. Repeat CT showed infarct (Figure 9.2). Decompression and duraplasty was done (Figure 9.3). The patient as discharged as dependent.

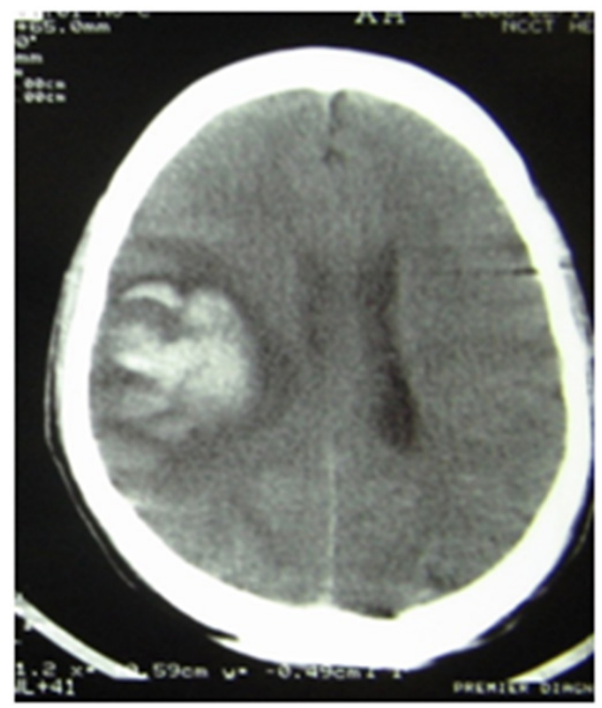

Figure 9.I CT scan showing right temporo-parietal hemorrhage. 


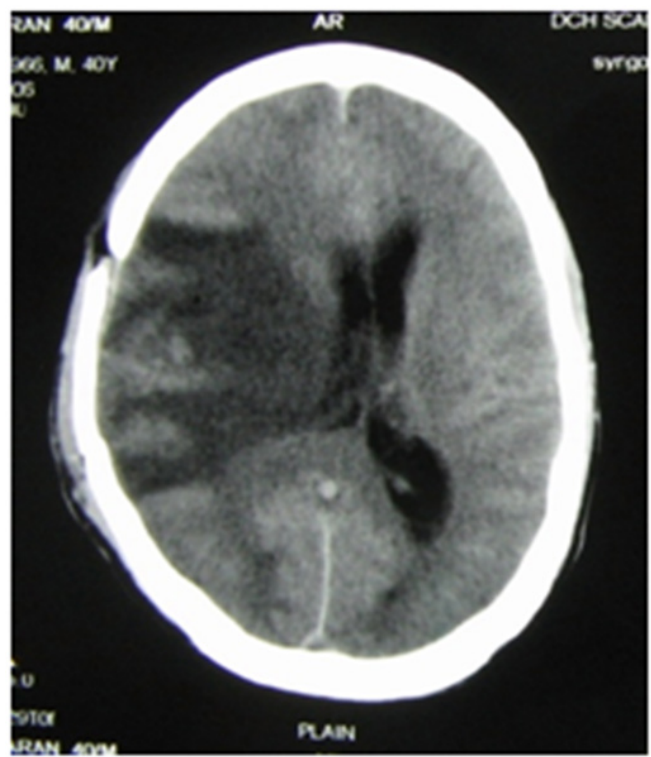

Figure 9.2 Repeat CT showing infarct.

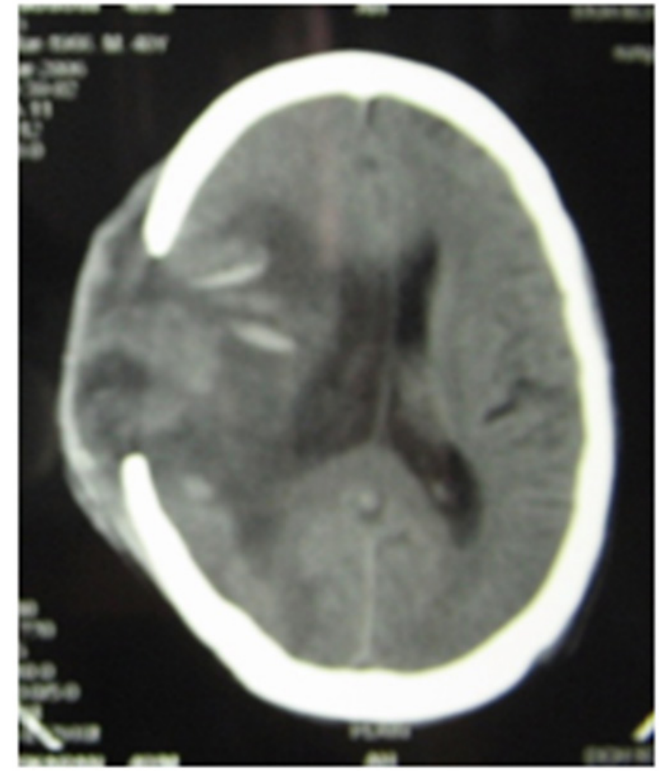

Figure 9.3 Postoperative CT following decompression and duraplasty.

\section{Discussion}

This study demonstrates the surgical outcome among patients with ICH following early surgery. It was found that surgery has best results among patients who are young and those with lesional or lobar hemorrhage. Adequate surgical decompression and intensive medical therapy is also necessary for favourable outcomes.

The mortality rate among the selected patients was $40 \%$, much lower than in earlier surgical series, where it is documented as $56 \%{ }^{2}$ The survival rate among those with lesional hemorrhage (90\%) was much higher than those with primary hemorrhage (47\%). Lobar hemorrhage was also found to be linked with better survival rates $(80 \%)$ than the basal ganglia hemorrhage $(41.6 \%)$.

It was also noted that GCS score $<8$ could predict a bad outcome than those with higher GCS scores. This was coherent with the findings from the STICH Trial. ${ }^{5}$ Younger patients had better results of survival than the older clients. Deep location of the bleed and Intra-ventricular hemorrhage was also found to be indicators of poor survival. Previous studies too have evidenced direct relation of the amount of blood in the ventricles to poor likelihood of survival. ${ }^{8,9}$

Adequate surgical decompression in the early stages and intensive multi-modal medical therapy could produce best results. As earlier published, decompression could prevent perihematomal edema, ischemia and also reduce intracranial pressure, thus reducing secondary injury or cascading harmful effects., ${ }^{2,6,10}$ Adequate ICU monitoring, fluid management, blood pressure control, ICP management, hemostatic therapy, temperature control, management of hyperglycemia and prevention of seizures are important measures to be undertaken for better prognosis of the patients. ${ }^{4,6}$

This single-centre study has limitations of comparative analysis with conservative management. The primary aim was to analyse the outcome rates in surgical therapy in the noncontroversial group. The strength of this study is that all the patients were examined, operated and followed up by a single investigator. The treatment strategies and modalities were well-equipped and provided by an experienced team of multi-modal physicians.

Furthermore, this study recommends future trials on advantages of minimally invasive clot evacuation of deep hematomas. Prospects of endoscopic and stereotactic drainage of clots too are yet to be researched upon.

\section{Conclusion}

Early surgery combined with multimodality medical management has definite positive role in the treatment of patients with spontaneous ICH. Lesional and lobar hematomas of young patients in good G.C.S. score have good outcome.

\section{Acknowledgments}

None.

\section{Conflicts of interest}

None.

\section{References}

1. Gregson BA, Mendelow AD; STICH Investigators. International Variations in Surgical Practice for Spontaneous Intracerebral Hemorrhage. Stroke. 2003;34(11):2593-2597.

2. Yilmaz C, Kabatas S, Gulsen S, et al. Spontaneous supratentorial intracerebral hemorrhage: Does surgery benefit comatose patients? Ann Indian Acad Neurol. 201013(3):184-187.

3. Zuccarello M, Brott T, Derex L, et al. Early Surgical Treatment for Supratentorial Intracerebral Hemorrhage A Randomized Feasibility Study. Stroke. 1999;30(9):1833-1899.

4. Rincon F, Mayer SA. Clinical review: Critical care management of spontaneous intracerebral hemorrhage. Crit Care. 2008;12(6):237.

5. Mendelow AD, Gregson BA, Rowan EN, et al. Early surgery versus initial conservative treatment in patients with spontaneous supratentorial lobar intracerebral haematomas (STICH II): a randomised trial. Lancet. 2013;382(9890):397-408

6. Sahni R, Weinberger J. Management of intracerebral hemorrhage. Vasc Health Risk Manag. 2007;3(5):701-709.

7. Broderick JP. The STICH trial what does it tell us and where do we go from here? Stroke. 2005;6(7):1619-1620. 
8. Hanley DF. Intraventricular hemorrhage severity factor and treatment target in spontaneous intracerebral hemorrhage. Stroke. 2009;40(4):1533-1538.

9. Bhattathiri PS, Gregson B, Prasad KS, et al. STICH Investigators. Intraventricular hemorrhage and hydrocephalus after spontaneous intracerebral hemorrhage: results from the STICH trial. Acta Neurochir Suppl. 2006;96:65-68.
10. Qureshi AI, Mendelow AD, Hanley DF. Intracerebral haemorrhage. Lancet. 2009;373(9675):1632-1644. 